\title{
Preparation, Characterization and comparative Temperature dependent Electrical Properties of Polythiophene \& its Nanocomposites using Carbon Nanotubes
}

\author{
Vikas $\operatorname{Sen}^{1} \&$ D.C. Tiwari ${ }^{2}$ \\ ${ }^{1}$ (Engineering, Manav Bharti University, India) \\ ${ }^{2}$ (Physics, Jiwaji University, India)
}

\begin{abstract}
The fabrication, characterization and electric measurement of Polythiophene and Carbon Nanotube (CNT) composite is described. The Polythiophene (PTh) and Polythiophene/ Multiwalled Carbon Nanotube (PTh/MWCNT) composite are prepared chemically by oxidative Polymerization of Thiophene (Th). The formation of PTh and incorporation of CNTs into PTh are confirmed by Fourier Transform - Infra Red spectroscopy (FT-IR) and Energy Dispersive X-Ray analysis (EDAX) analysis. Scanning Electron Microscopic (SEM) analysis revealed the formation of Polymer/CNT composite. SEM image of PTh shows a leaf like structure and image of PTh/MWCNT shows the incorporation of MWCNT into the PTh flakes. Atomic force microscopic (AFM) analysis shows the morphology of PTh and PTh/MWCNT. The structural features of Nanotube-Polymer association are observed by AFM studies. The electrical behavior of prepared samples is studied by measuring I-V characteristics at varying temperature. Conductivity shows a linear dependence on temperature and is also higher for PTh/MWCNT.
\end{abstract}

Keywords - Carbon nanotubes, Polythiophene \& conductivity.

\section{INTRODUCTION}

Thiophene based materials have emerged as one of the most promising materials due to the ease in its synthesis, its environmental stability and the possibility to modify it with different chemical groups $[1,2]$. Polythiophene in its original state is a non-conducting material. However chemical treatment with oxidants or electrochemical oxidation followed by anion intercalation can result in the formation of a conducting Polythiophene.

Carbon nanotubes (CNTs) have attracted a great deal of interest because of their unique properties. Multi-walled carbon nanotubes (MWCNTs) have been used in composite materials to improve electrical properties while reinforcing the mechanical performance of the composites [3-8].

In some special applications, such as space explorations, high-performance lightweight structural materials are required, and they can be developed by adding CNTs to polymers or other matrix materials. The superior properties of CNTs offer exciting opportunities for new composites. NASA has invested large sums of money to develop carbon nanotubes-based composites for applications such as the Mars mission [9].

Here in this paper we report the comparative electric study of polymer material and its nanocomposite using Carbon Nanotubes at different temperature ranges for further applications.

\section{II.1. MATERIAL}

\section{EXPERIMENTAL DETAILS}

Thiophene (Th) (Merck) is double distilled prior to use. Anhydrous Ferric chloride $\left(\mathrm{FeCl}_{3}\right)$ is purchased from Ranbaxy and used without further purification. Multiwalled carbon nanotubes (MWCNTs) are purchased from Aldrich.

\section{II.2. SYNTHESIS OF PTH/MWCNT COMPOSITE}

The synthesis of PTh is done by taking known amount of anhydrous $\mathrm{FeCl}_{3}(1 \mathrm{M})$ and this solution is added to $25 \mathrm{ml}$ of chloroform $\left(\mathrm{CHCl}_{3}\right)$ in a conical flask. To this solution, thiophene monomer $(1 \mathrm{M})$ is dissolved in $25 \mathrm{ml}$ of $\mathrm{CHCl}_{3}$ and is added to the conical flask drop wise using a burette. The reaction mixture is stirred continuously with magnetic stirrer at $0^{\circ} \mathrm{C}$ under controlled temperature conditions and later allowed to reach the room temperature with continuous stirring for 1 hour.

The polymer mixture is diluted with $\mathrm{CHCl}_{3}$ and filtered. The sample material collected by filtration is then washed several times with methanol and double distilled water for removing the unreacted oxidants and thiophene monomer until the solvent remains colorless. Finally, this Polymer material is dried in a vacuum chamber. PTh/MWCNT nano-composite is synthesized in a manner similar to that of PTh in the presence of MWCNTs by keeping ratio of PTh: CNT at 10:2. 


\section{II.3. Preperation Of Polymer Disk}

The prepared materials namely PTh and PTh/MWCNT are taken in equal amount to form their pellets by subjecting to pressure at $2000 \mathrm{lbs} / \mathrm{in}^{2}$ by hydraulic press (Spectra lab Model SL-89) for few minutes. The two pellets are then used for electrical measurements.

\section{MEASUREMENTS}

Electrical measurements are taken by connecting D.C. voltage power supply (Philips GP-4303D) and voltmeter (Kiethley-196) is placed parallel to the sample placed in sample holder and micro-ammeter (Simpson) is placed in series with the circuit.

\section{IV.1. FT-IR Analysis}

\section{RESULTS \& DISCUSSION}

The chemically prepared PTh and PTh/MWCNT are analyzed by FT-IR spectroscopy by making pellets with $\mathrm{KBr}$. The region from $500-1500 \mathrm{~cm}^{-1}$ is a finger print region for PTh and the peaks at 2800-3100 $\mathrm{cm}^{-1}$ are low intensity peaks attributed to aromatic $\mathrm{C}-\mathrm{H}$ stretching vibrations and $\mathrm{C}=\mathrm{C}$ characteristic band $\left(1652.66 \mathrm{~cm}^{-1}\right)$. The peak at $787.80 \mathrm{~cm}^{-1}$ is usually ascribed to the $\mathrm{C}-\mathrm{H}$ out-of-plane deformation mode, whereas other peaks in this region are attributed to the ring stretching, and $\mathrm{C}-\mathrm{H}$ in-plane deformation modes $[10,11]$. The C-S bending mode has been identified at approximately $694.13 \mathrm{~cm}^{-1}$ and indicates the presence of a thiophene monomer [12]. The PTh/MWCNT composite shows almost same position of the peaks in a range of 600-3200 $\mathrm{cm}^{-1}$. The $\mathrm{C}-\mathrm{H}$ stretching vibrations $\left(2800-3100 \mathrm{~cm}^{-1}\right)$ and $\mathrm{C}=\mathrm{C}$ characteristic peaks $\left(1647.52 \mathrm{~cm}^{-1}\right)$ can be identified almost in the same range respectively. The ring stretching and deformation modes are having a minor shift in the peaks because of polaron/ $\pi$ transition interaction between the PTh and MWCNT surface.

\section{IV.2. SEM-EDAX ANALYSIS}

The scanning electron microscopy Fig.1 shows leaf like structure of PTh while Fig. 2 shows the incorporation of MWCNTs into the PTh leafs. The SEM analysis revealed the interaction of MWCNTs with Polymer matrix because the presence of a random network of interconnected bundles of MWCNT, packed underneath the polymer confirm formation of polymer nanocomposite. Elemental EDAX analysis shows the atomic and weight percentage of PTh and PTh/MWCNT. The Wt \% of carbon in case of PTh is 56.78 and At\% is 78.38 as shown in Table 1 while in case of PTh/MWCNT the Wt\% and At\% of both increases as shown in Table 2 which gives an idea that increase in percentage of carbon is only due to the presence of MWCNTs. As $\mathrm{FeCl}_{3}$ is used as an oxidant in polymerization and due to that traces of iron are left after washing.

\section{IV.3. AFM ANALYSIS}

The morphology of PTh and PTh/MWCNT samples are investigated using a AFM analysis. Fig. 3 and Fig. 4 present a 3-D image of an AFM scan of the surface of PTh and PTh/MWCNT having nodular structure. In case of PTh/MWCNT nodular size is higher than PTh. Nodular structure is associated with the highly doped conducting samples in which nodules represent dopant rich highly conductive area [13]. In our composite we find the same nodular structures indicating that the polymer growth process is not affected significantly by the insertion of nanotubes. The insertion of thin bundles of these MWCNTs into the polymer matrix allows the formation of large shapeless nodules increasing the conductivity of the sample.

In case of PTh the particle size is of $338 \mathrm{~nm}$ and the roughness of sample is of $57 \mathrm{~nm}$ but for $\mathrm{PTh} / \mathrm{MWCNT}$ nanocomposite the roughness reduces to $13 \mathrm{~nm}$ as the particle size of MWCNTs is $61 \mathrm{~nm}$ which is very small and hence it acts as filler respectively.

MWCNTs and Thiophene (Th) monomer are polymerized simultaneously and it is found that the PTh has grown across the MWCNT, due to nano dimensions of CNTs it leads to smoothening of resulted $\mathrm{PTh} / \mathrm{MWCNT}$ nanocomposite. As we know that the nanomaterials they occupy interfacial sights, in a way they act like a filler material. The unpaired lattice defects on the surface of CNTs act as active centers for the interaction of PTh molecular chains on the surface of CNTs. The reduced roughness and particle size increases the polymer conductivity.

\section{IV.4. ELECTRICAL MEASUREMENT}

In order to make electronic measurements of PTh and PTh/MWCNT nanocomposite the I-V characteristics are measured at four different temperature ranges and comparison is seen between both the materials, shown in Fig. 5 and Fig. 6.

In case of PTh the voltage is varied from 10 to $50 \mathrm{~V}$ in a step wise manner and the current is measured at varying temperature. I-V characteristics show almost linear relationship and it is noticed that the value of current increased for every applied voltage with increase in temperature. The electrical behavior of Polythiophene sample is poor semiconducting. 
However, in case of PTh/MWCNT nanocomposite the voltage is applied from 10 to $80 \mathrm{mV}$ and large variation in current is noticed in comparison to PTh and in a similar manner there is also increase in current for every applied voltage with increase in temperature. The incorporation of MWCNTs into the PTh matrix converts its electrical behavior from poor semiconducting to semiconducting and increase in value of current with increase in temperature is due to thermal expansion and rapid increase in ion mobility.

Table1: Elemental composition of PTh

\begin{tabular}{cll}
\hline Elements & $\mathrm{Wt} \%$ & At\% \\
\hline $\boldsymbol{C K}$ & 56.78 & 78.38 \\
SK & 38.10 & 19.70 \\
$\boldsymbol{C l K}$ & 02.33 & 01.09 \\
$\boldsymbol{F e} \boldsymbol{K}$ & 02.78 & 00.83 \\
\hline
\end{tabular}

Table2: Elemental composition of PTh/MWCNT

\begin{tabular}{cll}
\hline Elements & $\mathrm{Wt} \%$ & $\mathrm{At} \%$ \\
\hline $\boldsymbol{C K}$ & 71.25 & 87.01 \\
$\boldsymbol{S K}$ & 27.54 & 12.60 \\
$\boldsymbol{C l K}$ & 00.45 & 00.18 \\
$\boldsymbol{F e} \boldsymbol{K}$ & 00.76 & 00.20 \\
\hline
\end{tabular}

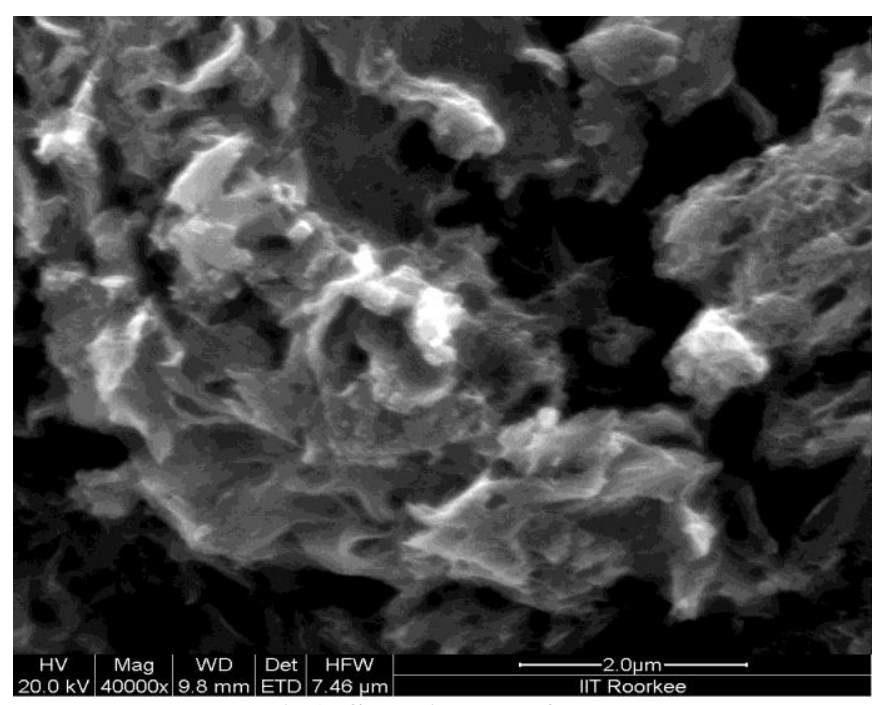

Fig1. SEM image of PTh

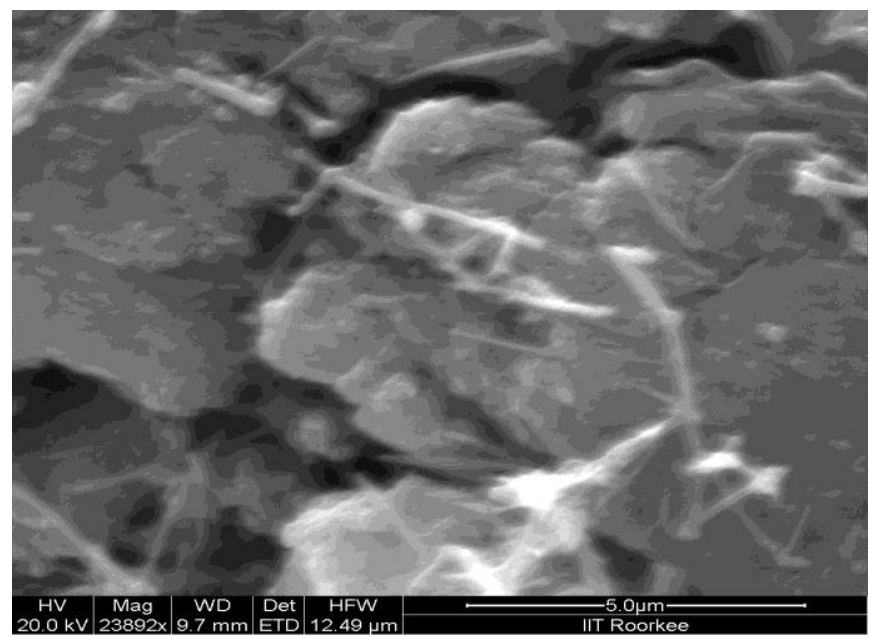

Fig2. SEM image of PTh/MWCNT 


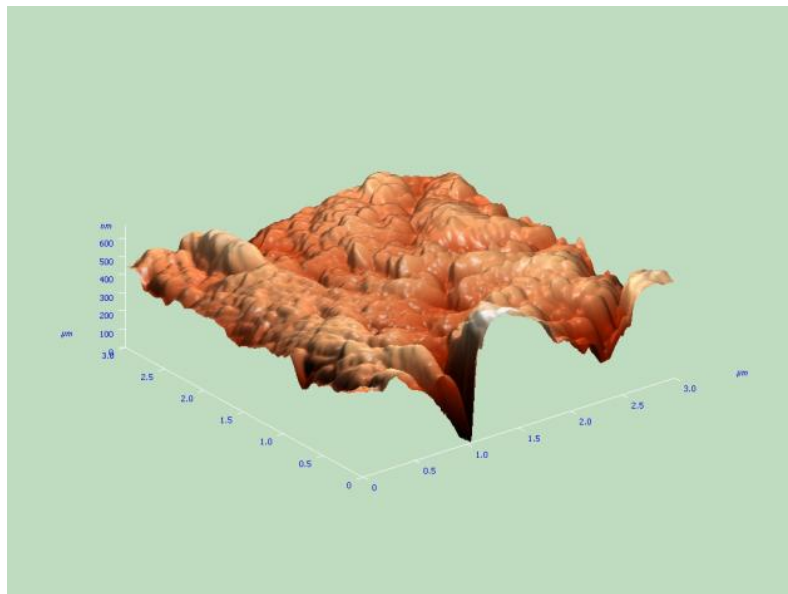

Fig.3 3-D AFM image of PTh

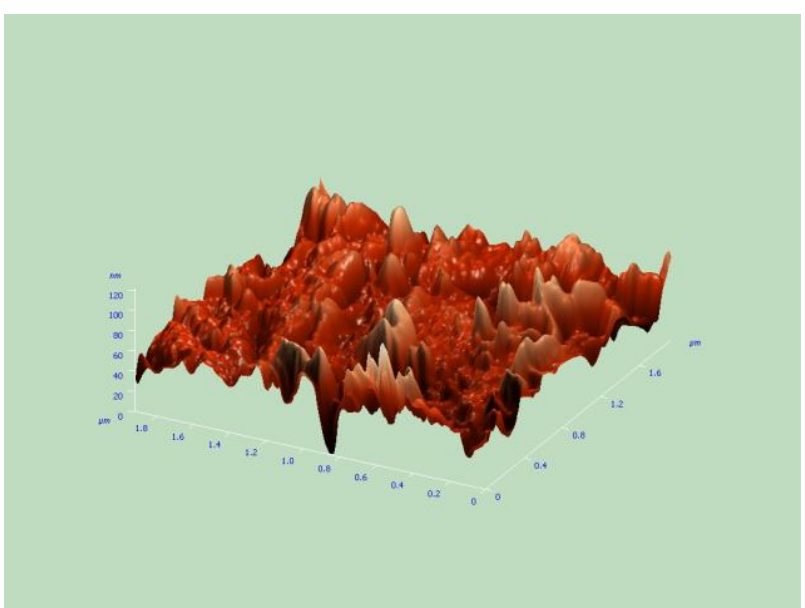

Fig.4 3-D AFM image of PTh/MWCNT

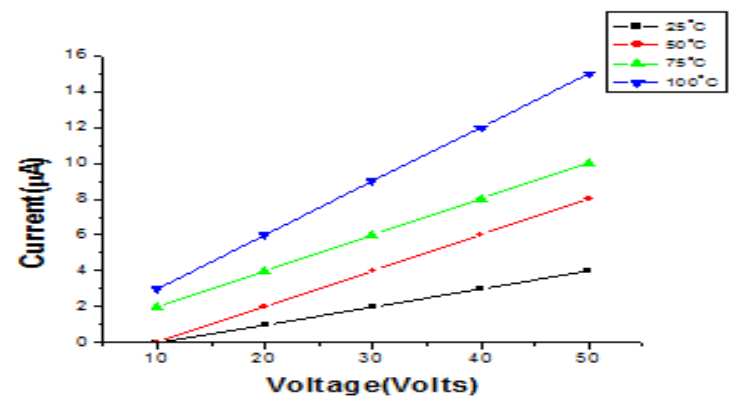

Fig.5 I-V parameters of PTh at varying Temperature

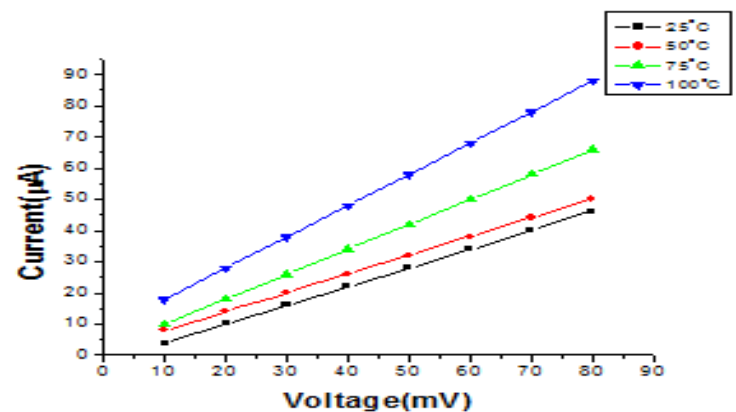

Fig.6 I-V parameters of PTh/MWCNT nanocomposite at varying Temperature 


\section{CONCLUSION}

In the present study we synthesized Polythiophene/ MWCNT nanocomposite by using a monomer that undergoes oxidative polymerization in the presence of Multiwalled carbon nanotubes. Following this chemical route, it is possible to generate polymer-based composites in which the nanotubes are not simply mixed up with the polymer, but are also in intimate contact with the polymer through specific chemical interactions and this formation is confirmed by SEM images. The above described synthesis proves to be a feasible pathway for the fabrication of a very important class of materials based on nanotube/polymer systems.

The electrical measurements for both PTh and PTh/MWCNT are taken by analyzing I-V parameters. The electrical behavior of $\mathrm{PTh} / \mathrm{MWCNT}$ changes to semiconducting from basic poor semiconducting polythiophene. This drastic change increases the quality of material and creates an idea for large view point of applications in commercial and industrial use. . It is observed that incorporating MWCNTs into PTh modifies the electrical behavior and almost metallic type of behavior of these nanotubes gives rise to a conducting Polymer nanocomposite material. This increase in conductivity of polymer by incorporating MWCNTs can be used for solar cells, EM Shielding, metal oxide semiconductor devices etc.

\section{Acknowledgements}

This work is financially supported by Madhya Pradesh Council of Science and Technology (MPCST), Bhopal, India. Thanks to IIT Roorkee for characterizing the samples.

\section{REFERENCES}

[1]. I F Perepichka, D F Perepichka, H Meng, F Wudl, Adv.Mater. 17 (2005) 2281.

[2]. J Roncali, Chem. Rev. 92 (1992) 711.

[3]. A Allaoui, S Bai, H M Cheng, and J B Bai, Compos. Sci. Technol. 62 (2002) 1993

[4]. H Geng, R Rasen., B Zheng, H Shimoda, L Fleming, J Liu and O Zhou, Adv. Mater. 14 (2002) 1387.

[5]. Z Ounaies, C Park, K E Wiseb, E J Siochic and J.S. Harrisonc, Compos. Sci. Technol. 63 (2002) 1637.

[6]. $\quad$ S Cui, R Canet, A Derre, M Couzi and P Delhaes, Carbon. 41 (2003) 797.

[7]. M Cadek, J N Coleman, V Barron, K Hedicke and W J Blau, Appl. Phys. Lett. 81 (2002) 5123.

[8]. M A López Manchado, L Valentini, J Biagiotti, and J M Kenny, Carbon. 43 (2005) 1499.

[9]. M S Dresselhaus, G Dresslhous, P Avouris, 'Carbon Nanotubes: Synthesis, Structure, Properties and Application' (Springer, Berlin, Germany, 2001).

[10]. J Roncoli, Chem Rev. 92 (1992), 711.

[11]. N Toshima, S Hara, Prog Polym Sci. 20 (1995) 155.

[12]. Y A Udum, K Pekmez, A Yildiz, Eur Polym J. 41 (2005) 1136.

[13]. J N Barishi, R Stella, G M Spinks, G G Wallace, Electrochim Acta 46(4) (2000) 519. 